ESAIM: PROCEEDINGS AND SURVEYS, January 2015, Vol. 48, p. 321-340

N. Champagnat, T. Lelièvre, A. Nouy, Editors

\title{
NUMERICAL SIMULATION OF THE DYNAMICS OF SEDIMENTARY RIVER BEDS WITH A STOCHASTIC EXNER EQUATION
}

\author{
Emmanuel Audusse ${ }^{1,2}$, Sébastien Boyaval $^{3,4}$, Nicole Goutal $^{3,5}$, Magali Jodeau $^{5}$ \\ AND PhILIPPE UNG ${ }^{1,6}$
}

\begin{abstract}
At the scale of a river reach, the dynamics of the river bed is typically modelled by Exner equation (conservation of the solid mass) with an empirical solid flux of transported sediments, which is a simple deterministic algebraic formula function of $i$ ) the sediment physical characteristics (size and mass) and of ii) the averaged hydrodynamical description of the ambient water flow. This model has proved useful, in particular through numerical simulations, for hydraulic engineering purposes (like estimating the mass of sediments that is drained through an open dam). Though, the model is also coarse. And its applicability at various space and time scales remains a question of considerable interest for sedimentologists. In particular, physical experiments from the grain scale to the laboratory scale reveal important fluctuations of the solid flux in given hydrodynamical conditions.

This work is a preliminary study of the coupling of a stochastic Exner equation with a hydrodynamical model for large scales. (Stochastic models with a probabilistic solid flux are currently being investigated, but most often only from the viewpoint of theoretical physics at the grain scale.) We introduce a new stochastic Exner model and discuss it using numerical simulations in an appropriate test case.
\end{abstract}

Résumé. A l'échelle d'un bras de rivière, la dynamique du lit de la rivière est généralement modélisée par l'équation d'Exner (conservation de la masse solide) avec un flux solide empirique pour le transport de sédiments, flux défini par une formule algébrique et déterministe dépendant i) des caractéristiques physiques des sédiments (taille et masse) et ii) de la description hydraulique moyenne de l'écoulement local. Ce modèle s'est avéré utile, notamment à travers des simulations numériques, pour des applications en ingénierie hydraulique (par exemple, estimation de la masse de sédiments mobilisée lors d'une vidange de barrage). Néanmoins, le modèle est également grossier. Et son utilisation pour des échelles de temps et d'espace variées reste une question d'un intérêt considérable pour les sédimentologues. En particulier, les expériences physiques allant de l'échelle du grain à celle du laboratoire révèlent d'importantes fluctuations du flux solide sous des conditions hydrodynamiques données.

Ce travail est une étude préliminaire du couplage d'une équation d'Exner stochastique avec un modèle hydrodynamique pour les grandes échelles. (Des modèles stochastiques prenant en compte un flux solide probabiliste sont actuellement étudiés, mais le plus souvent seulement du point de vue de la physique théorique à l'échelle du grain.) On introduit un nouveau modèle d'Exner stochastique possible puis on le discute à l'aide de simulations numériques à travers un cas test approprié.

\footnotetext{
1 Team ANGE, INRIA-Paris-Rocquencourt

${ }^{2}$ LAGA, Université Paris XIII, France

${ }^{3}$ Université Paris Est, Laboratoire d'Hydraulique Saint-Venant (Ecole des Ponts ParisTech - EDF R\&D - CEREMA)

${ }^{4}$ INRIA Rocquencourt, France

${ }^{5}$ LNHE, EDF R\&D

${ }^{6}$ MAPMO, Université d'Orléans, France, e-mail: ung@math.cnrs.fr
}

(C) EDP Sciences, SMAI 2015 


\section{INTRODUCTION}

Sediments are particles that can be transported by rivers over large distances. Their transport, deposition or erosion, controls the form of rivers. Depending on the water discharge, the geology, the slope of the area and the supply of sediment, rivers exhibit braid, meander or straight patterns. The study of sediment dynamics is particularly useful to prevent the filling of hydroelectric reservoirs, but also to preserve water intakes in rivers. Furthermore, sediments are a major component defining the habitat of fishes.

Two different kinds of transport processes are usually distinguished for non-cohesive sediments [25] : (i) fine particles (typically smaller than $2 \mathrm{~mm}$ ) are mainly transported by suspension, contacts with the bed are then rare and particles can be found on the whole vertical of water depth ; (ii) larger particles are transported by bedload near the bed, by rolling or small jumps. This present work is focused on bedload transport.

A usual model to describe bedload is to write (i) a sediment mass conservation equation, the well-known Exner equation [14], and (ii) an empirical formula for the flux of sediments, experimentally validated in bedload conditions (moving sediments remain close to the bed).

Most numerical codes modelling the transport of sediments for engineering studies (that is at scales involving a river reach and many days) make use of these equations (for example Telemac-Sisyphe [27], or many other codes [38]...). They have correctly predicted useful information such as an approximation of the total mass of sediments transported during a flood. Among other examples, [13] shows that with a one dimensional sediment transport model, they achieve reasonably good results in reproducing the bed changes due to a large flood event on the Ha!Ha! River (Canada). In [45], the authors compare calculations of solid discharge of the Danube River with measurements from a bed-load sampler and show good agreement with the observations. Nevertheless this model remains definitely coarse.

Empirical formulae for the flux of sediments are simply written as algebraic functions of the locally-averaged hydrodynamical conditions above the river bed, since what happens under the river is obviously inaccessible in most practical situations. There are many different established formulae for different flow conditions and sediment sizes, which can still be only determined experimentally at a high cost. Furthermore, the model has proved unable so far to account for wave-like transient phenomena which have been actually observed in rivers at any scale: ripples, dunes...

In any case, because of both its advantages and limitations, Exner model is still attracting the attention of many modellers interested by the dynamics of a river bed. In fact, the very notion of solid mass flux (or discharge) in bedload conditions is still discussed, for it strongly depends on the time and space scales at which transport is modelled. Its stochastic features in particular are discussed. On the one hand, a probabilistic framework at the grain scale has indeed been used for a long time, but also more recently, to propose semiempirical deterministic bedload formulae at large scales $[12,47]$ after direct averaging of the stochastic solid discharge. On the other hand, the solid discharge also exhibits stochastic features at larger scales in laboratory experimental measures [22,41], see for instance Figure 1, although the probability distributions reconstructed from the measurements may depend on the sampling $[9,22]$. Moreover, the idea has also recently emerged that some persistent forms observed at the surface of river beds may result from the nonlinear interactions of small-scale features that should be modelled through the stochastic fluctuations, see e.g. [3,16,30,34]. Finally, it appears natural to ask whether the solid discharge should still be considered stochastic in the models used for engineering studies, and then on which ground.

In this work, we suggest one possible stochastic Exner model where the solid mass flux is directly defined as a random variable with a probability distribution in keeping with the experimental measurements available in the literature $[22,41]$. Then, we numerically investigate this new model in a standard benchmark test case for sediment transport, and we discuss some mathematical problems that naturally arise during the construction of such probabilistic models. Our goal is to evaluate the potential of new probabilistic models as improvements of the existing codes using Exner model at the scale of a river reach. We do not discuss the physics at the grain scale that could naturally define a stochastic solid discharge, but we demonstrate the need for an adequate mathematical framework before new probabilistic models can be coupled to hydrodynamic models at large 


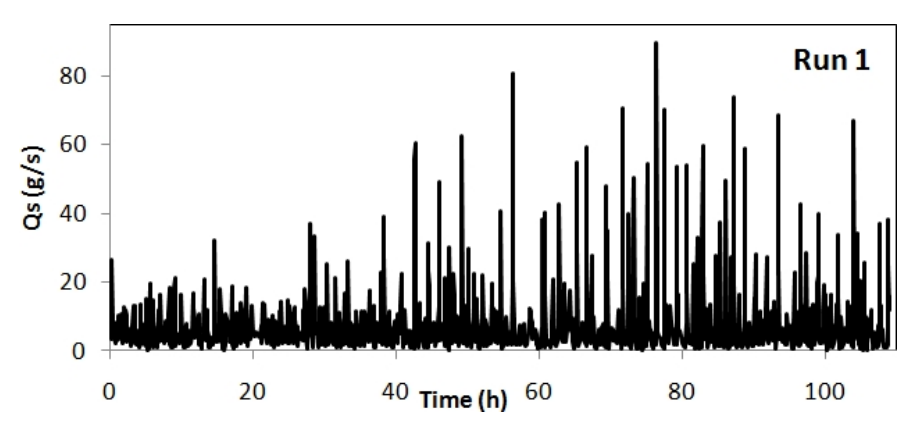

(A) Time variation of solid discharge.

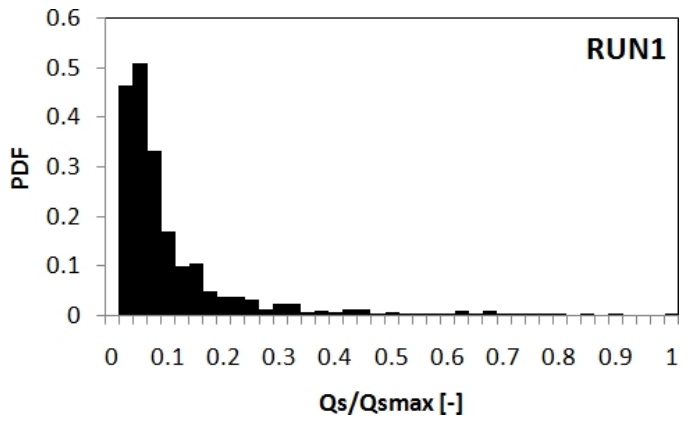

(B) Distribution of solid discharge from figure 1a.

FigURE 1. Flume experiment of bedload transport on high slopes, data kindly provided by A. Recking. Experiment was performed as described in [41] .

scales. Much more experimental data (i.e. two-point correlations in space at least) seem necessary in order to correctly achieve that latter goal.

\section{EXNER MODEL}

\subsection{The standard Exner model}

Let us denote by $x \in \mathbb{R}$ the abscissa along a one-dimensional (1D) rectilinear infinite river. Exner equation is a local mass balance of sediments under a river bed that is modelled as a graph $x \rightarrow B(t, x)$ for all times $t \geq 0$

$$
\phi_{s} \partial_{t} B=-\partial_{x} Q_{s}
$$

on assuming that the volume fraction $\phi_{s}$ of the solid phase remains constant under the river bed. The model is closed after precising the $1 \mathrm{D}$ flux of sediments $Q_{s}\left(\right.$ in $\left.[m]^{2} \cdot[s]^{-1}\right)$ flowing through a section close to the river bed (bedload transport). Closure is a very complicated problem in general. River beds are polydisperse (fluidsaturated) granular suspensions with a very complicated rheology. However, one can create flow conditions in laboratory flume experiments such that $Q_{s}$ can be measured.

In experimental settings where sediments are heavy and only move by saltation, rolling and sliding motions close to the river bed $[15,39]$, one retrieves a non-dimensional solid mass flux $Q_{s}^{\star}$ after comparing the data with

$$
Q_{s}=\sqrt{\frac{g\left(\rho_{s}-\rho\right) d^{3}}{\rho}} Q_{s}^{\star}\left(\tau^{\star} ; \tau_{c}^{\star}\right) \frac{\tau^{\star}}{\left|\tau^{\star}\right|}
$$

where $g$ is the gravity constant, $\rho_{s}, \rho$ are respectively the mass densities of the solid and fluid phases, $d$ is a typical grain diameter for sediments. For instance, Meyer-Peter and Müller [33] (MPM) have proposed

$$
Q_{s}^{\star}=8\left(\left|\tau^{\star}\right|-\tau_{c}^{\star}\right)_{+}^{\frac{3}{2}}
$$

for gravel, that is a function of the dimensionless shear stress term also called the Shields parameter [43]

$$
\tau^{\star}=\tau /\left(g\left(\rho_{s}-\rho\right) d\right)
$$

where $\tau$ is the shear stress exerted by the flow on particles at the surface of the river bed (in $\left.[k g][m]^{-1}[s]^{-2} \equiv P a\right)$, and $\tau_{c}^{\star}$ is a critical value for the initiation of motion (tabulated for various grain types). Shields [43] indeed noted that sediment transport occurs when the bottom shear stress just exceeds the threshold of incipient motion $\tau_{c}$. 
In uniform flow conditions, the shear stress $\tau$ can be directly computed as a local function of the river bed materials characteristics and of the hydrodynamic conditions above the river bed, namely the water depth $H$ and a "(bed-)shear" velocity $U^{\star}$. In particular, following the Darcy-Weisbach law that says that the specific energy loss in uniform flow conditions is proportional to some mean velocity $|U|^{2}$, we use the Manning formula

$$
\tau=\rho g H \frac{Q|Q|}{H^{2} K_{s}^{2} R_{h}^{4 / 3}},
$$

where, in the particular case of a rectangular channel with width $l$, the hydraulic radius $R_{h}$ reads

$$
R_{h}=\frac{l H}{l+2 H} .
$$

For numerical simulations, the dynamics of the whole river (sediments plus water) is finally computed from a model coupling (1) to the hydrodynamics of the free-surface water flow over the given bed $B$. Typically, for application to large space scales where Exner equation is complemented with MPM relation (2), it is often assumed that a coarse hydrodynamical model like the Saint-Venant nonlinear shallow-water equations

$$
\left\{\begin{array}{l}
\partial_{t} H+\partial_{x} Q=0 \\
\partial_{t} Q+\partial_{x}\left(Q^{2} / H+g H^{2} / 2\right)=-g H \partial_{x} B-\frac{\tau}{\rho}
\end{array}\right.
$$

modelling a hydrostatic free-surface shallow flow of water with depth $H(t, x)$ and discharge $Q(t, x)$ above a river bed with small-amplitude variations in the longitudinal direction. Note that the friction term in (4) was defined in (3) and the mean velocity $U$ is defined as the ratio $Q / H$ so the system (1)-(4) with relations (2)-(3) is closed.

\subsection{A new stochastic Exner model}

Let us now propose a new stochastic Exner model. We shall directly introduce some "noise" in the model used at large scale. That is, some variables of the standard Exner model are now made random with a prescribed probability distribution in keeping with experimental measures in stationary conditions. Notice that this requires in particular that the solid discharge at the grain-scale (experimentally measurable) has not only a well-defined probability distribution on each finite space-time intervals of a given scale, but also that the joint probability distribution of various non-overlapping intervals is also well-defined. We are aware that such a scale is not yet fully identified, see e.g. [9], but current investigations show promising results [22], although joint probabilities for instance are not yet accessible. Anyway, assuming a separation of scales is natural when building a mathematical model (here, between microscopic processes at the grain scale and macroscopic transport at Exner scale), and we rely, as in most models, on the assumption of "locally uniform hydrodynamic flow conditions".

To start with, we use one common definition of the solid mass flux $Q_{s}$, see e.g. [17]:

$$
Q_{s}=d \tilde{n} V
$$

where $d$ is the particle diameter, $\tilde{n}$ is the number of saltating particles per space unit and $V$ their longitudinal velocity. Then, with Bagnold scaling [8]

$$
V=\sqrt{\frac{g\left(\rho_{s}-\rho\right) d}{\rho}} a\left(\sqrt{\tau^{\star}}-\sqrt{\tau_{c}^{\star}}\right)
$$

and the local equilibrium condition $\tilde{n} \propto\left(\tau^{\star}-\tau_{c}^{\star}\right)$, one retrieves the usual Meyer-Peter and Müller formula (2) once the empirical "activity" number $a$ is fitted to 8 from the empirical average of experimental measurements with gravel in bedload conditions. Now, experimentally measured time-sequences have also brought out that 
the activity number $a$ is in fact exponentially-distributed pointwise-in-space, see e.g. [32]. Assuming an ergodic underlying process, we thus suggest to use

$$
Q_{s}^{\star}=A\left(\left|\tau^{\star}\right|-\tau_{c}^{\star}\right)_{+}^{\frac{3}{2}}
$$

with a random field $A$ exponentially-distributed pointwise in space and time with mean 8

$$
\mathbb{P}(A(t, x) \leq \bar{A})=\int_{0}^{\bar{A}} 8 e^{-8 A} d A=1-e^{-8 \bar{A}}
$$

Note that this has also the meaning of an exponentially distributed travel distance $\lambda$ for individual particles after rewriting $n V=E \lambda$ with a (deterministic) pick-up rate $E$. Furthermore, since we would like our new model to be an enriched version of the standard Exner model and to yield back the standard Meyer-Peter and Müller formula for gravel in the "mean-field" approximation $A(t, x) \approx \mathbb{E}_{\mathbb{P}}(A)$, it was natural indeed to choose $\mathbb{E}_{\mathbb{P}}(A)=8$. Though, the model is not fully defined yet. In particular, it is not clear how to define the dependence of the stochastic field $A(t, x)$ on $t$ and $x$.

In this project, we next explore numerically (i.e. at a discrete level) the implications of the "simple" choice where all the random variables $A(t, x)$ at different $t$ and $x$ are chosen independent one-another, when Exner model is coupled to a hydrodynamical model for large scales (as it is usual in practice), and we explain why, in that frame, such a simple choice turns out to be a problem from the physical viewpoint.

Note that several recent works have already embraced the prospect of building a stochastic model of Exnertype. For instance, in [30], a white noise in time and space has been added to a slightly modified closure of (1) given historically by Exner himself [14], when $Q_{s}$ is an algebraic function of $B$ and also of the slope $\partial_{x} B($ then $(1)$ becomes a closed equation). The model potentially describes complex bed evolutions, but the postulated solid discharge happens not to be verified in most flow conditions.

Using a microscopic model at the grain scale, and focusing on small variations around a planar bed at rest, [2] computes the distribution of $-\partial_{x} Q_{s}$ in uniform flow conditions (i.e. insensitive to a Lagrangian vs. Eulerian description) as a balance between deposition and erosion processes (see also [40] for details about this reformulation). The deposition and erosion processes are chosen as simple algebraic functions of the total number $\tilde{N}$ of particles in a control volume, with time variations proportional to those of the local bed elevation. In other words, Exner equation is reinterpreted as the master equation of a birth-death process followed by $\tilde{N}$, using the deposition and erosion processes as birth and death rates. In a formal large- $\tilde{N}$ limit [3], the pointwise probability distribution of the solid flux is Gaussian and not in keeping with experimental observations [32]. Note also that in this model, the coupling with the surrounding (uniform) hydrodynamics is through the deposition and erosion processes, which require a given particles velocity. The extension to non-uniform flow conditions (and thereby the coupling with hydrodynamics at large scales) is not straightforward.

In the series of papers [18-21,42], a kinetic approach is used in order to derive Exner equation, from the evolution equation of the probability density of particles possessing some "activity" $\gamma$ at position $x$ and time $t$, the time-derivative of that density supposedly being directly proportional to the time-derivative of the bed elevation. This yields a reinterpretation of Exner equation as a nonlinear advection-diffusion equation for the bed elevation, and consequently includes the possibility of a diffusive behaviour that is sometimes observed in experiments (depending on the flows and on the sampling-time of measures). However, this kinetic approach remains rather formal, and does not allow for a precise computation of the advective and diffusive coefficients from an explicit stochastic process satisfied by the particles activity. The coefficients are evaluated empirically. In fact, the same exponential distribution observed in [32] for the longitudinal particles velocity is invoked.

Note that the ability of the approaches above at describing a full erosion/deposition scenario better than the standard Exner model, especially when coupled with a hydrodynamic model that lets the (locally uniform) flow conditions evolve, has not been tested yet. This is exactly what we have in mind here, with our model. Of course, this would be difficult with the model of [30], especially because, whatever the solid discharge is used in 
Exner equation, adding a source term violates the principle of solid mass conservation. And a computation of the local solid discharge with $[2,3]$ first requires to extend the model to non-uniform flow conditions. Last, with a small diffusive term, the kinetic approach of [18-21,42] cannot improve on standard Exner model: it is not a new equation and it still requires empirical fitting. Contrary to the latter interpretation of Exner equation starting with a kinetic framework and ending with a fully deterministic closure at the hydrodynamic scale, our model lets the coupled hydrodynamic depend stochastically (through the coupling) on the random particles activity. This is potentially richer, and may allow some "variability in the bedform geometry [to result] from some randomness in sediment flux" [3]. Of course, this is also potentially dangerous, and the first relevant question to ask is: does such a fully coupled model contain at least the uniform flow conditions used for the matching with experimental measures? That question still needs to be precised and shall next be investigated numerically. The notion of uniform flow conditions in particular may have to be understood only "in the mean", while the mean behaviour is in fact quite complicated because of the nonlinearities.

\section{A NUMERICAL DisCUSSION OF THE STOCHASTIC MODEL}

\subsection{Finite-Volume discretization of Saint-Venant-Exner model}

Recall Saint-Venant-Exner model with periodic boundary conditions on the unit cell $\mathcal{S}=\{x \in[0,1)\}$ of $\mathbb{R} / \mathbb{Z}$

$$
\left\{\begin{array}{l}
\partial_{t} H+\partial_{x} Q=0 \\
\partial_{t} Q+\partial_{x}\left(\frac{Q^{2}}{H}+\frac{g H^{2}}{2}\right)+g H \partial_{x} B=-\frac{\tau}{\rho} \\
\partial_{t} B+\partial_{x} Q_{s}=0
\end{array}\right.
$$

where $g$ represents the gravitational acceleration, $H(t, x)$ is the water height, $Q(t, x)$ the discharge and $B(t, x)$ the topography. The solid transport flux $Q_{s}(t, x)$ is defined by the stochastic Meyer-Peter-Müller formula (5) and the friction term is given by relation (3).

We now describe how we numerically solve the Saint-Venant-Exner system (6). Let us first mention some of the recent works $[4,10,24]$ devoted to the derivation of finite volume methods to handle system (6) at once. Here we do not adopt this strategy and apply a splitting method on a staggered grid in a finite volume - finite difference spirit. Indeed it is easier to preserve a discrete steady state on a constant slope in this way and it also allows us to clearly separate the influence of the stochastic term on the bottom topography on one hand and on the fluid quantities on the other hand.

Let us first introduce some notations. We define two meshes of the domain. We first define a number of semicells equal to $2 N_{x}$ denoted $C_{i, i+1 / 2}$ and define the cells $C_{i}=C_{i-1 / 2, i} \cup C_{i, i+1 / 2}$ and $C_{i+1 / 2}=C_{i, i+1 / 2} \cup C_{i+1 / 2, i+1}$ for the two meshes, $i=0, \ldots, N_{x}-1$. For simplicity we consider uniform meshes and denote the space step $\Delta x$. We define the time step $\Delta t^{n}$ later on. The fluid quantities will be defined on cells $C_{i}$ and the bottom topography on cells $C_{i+1 / 2}$.

Then starting from initial quantities defined as the mean values of the initial datas on meshes $C_{i}$ or $C_{i+1 / 2}$

$$
H_{i}^{0}=\int_{C_{i}} h^{0}(x) d x, \quad U_{i}^{0}=\int_{C_{i}} u^{0}(x) d x, \quad B_{i+1 / 2}^{0}=\int_{C_{i+1 / 2}} b^{0}(x) d x
$$

we compute at each time step the numerical solution in two steps

(i) first, we compute approximate values for the fluid quantities $\tilde{W}=(H, Q)^{T}$ by classical finite volume formulas on cells $C_{i}$

$$
\tilde{W}_{i}^{n+1}=\tilde{W}_{i}^{n}-\frac{\Delta t^{n}}{\Delta x}\left(F_{i+1 / 2}^{n}-F_{i-1 / 2}^{n}\right)+\frac{\Delta t^{n}}{\Delta x} \tilde{S}\left(\tilde{W}_{i}^{n}, B_{i-1 / 2}^{n}, B_{i+1 / 2}^{n}\right),
$$


where $F_{i+1 / 2}$ is the numerical flux defined by the Rusanov formula in this present case,

$$
F_{i+1 / 2}=F\left(\tilde{W}_{i}, \tilde{W}_{i+1}\right)=\frac{F\left(\tilde{W}_{i}\right)+F\left(\tilde{W}_{i+1}\right)}{2}-c \frac{\tilde{W}_{i+1}-\tilde{W}_{i}}{2}
$$

with $F(\tilde{W})=\left(Q, \frac{Q^{2}}{H}+\frac{g H^{2}}{2}\right)^{T}, c=\max \left(\left|U_{i}\right|+\sqrt{g H_{i}},\left|U_{i+1}\right|+\sqrt{g H_{i+1}}\right)$ and $\tilde{S}\left(\tilde{W}_{i}^{n}, \tilde{W}_{i+1}^{n}\right)$ is a discrete source term written as

$$
\tilde{S}\left(\tilde{W}_{i}^{n}, B_{i-1 / 2}^{n}, B_{i+1 / 2}^{n}\right)=\left(\begin{array}{c}
0 \\
g H_{i}^{n}\left(B_{i+1 / 2}^{n}-B_{i-1 / 2}^{n}\right)
\end{array}\right)
$$

(ii) second, we compute approximate values for the bottom topography by finite difference formulas

$$
B_{i+1 / 2}^{n+1}=B_{i+1 / 2}^{n}-\frac{\Delta t^{n}}{\Delta x}\left(Q_{s}\left(H_{i+1}^{n}, U_{i+1}^{n}\right)-Q_{s}\left(H_{i}^{n}, U_{i}^{n}\right)\right),
$$

Since we work on the torus $\mathcal{T}^{1}$, we have to deal with periodic limit conditions. For the fluid mesh, we add two ghost cells $C_{-1}$ and $C_{N_{x}}$ where we define the periodic limit conditions as follows

$$
\begin{cases}H_{-1}=H_{N_{x}-1}, & Q_{-1}=Q_{N_{x}-1}, \\ H_{N_{x}}=H_{0}, & Q_{N_{x}}=Q_{0}\end{cases}
$$

For the bottom topography, we similarly define two ghost cells

$$
\left\{\begin{array}{l}
B_{-1 / 2}=B_{N_{x}-1 / 2} \\
B_{N_{x}+1 / 2}=B_{+1 / 2}
\end{array}\right.
$$

Note however that $B_{N_{x}+1 / 2}$ is never actually used in the numerical scheme above because of the discretization choices (explicit fluxes and centered source terms).

We then have to define the time step. It has to satisfy the CFL condition for the Saint-Venant system

$$
\Delta t^{n} \leq \frac{\Delta x}{\max _{i=1, \cdots, N_{x}}\left(\left|U_{i}^{n}\right|+\sqrt{g H_{i}^{n}}\right)}
$$

Let us recall that our goal is to perform Monte-Carlo simulations and then to run many realizations. Moreover we also would like to consider different space steps $\Delta x \in\{0.5,0.25,0.125,0.0625,0.03125\}$. To make the comparison between the results easier, we then choose a quite restrictive time step that will satisfy the CFL condition in any case. The uniform choice $\Delta t=10^{-2} s$ is made for all computations in the present study.

\subsection{Monte-Carlo simulations and numerical results}

Let us first mention that all our simulations for the semi-empirical stochastic model are close to a deterministic case that is well understood: stationary uniform flow. Moreover we concentrate on torrential regime where the experiment of Recking [41] exhibits important fluctuations in solid discharge $Q_{s}$. Then we first describe the uniform deterministic solution, we second describe the stochastic perturbation of the solid flux and third we comment the results of the Monte-Carlo simulations.

\subsubsection{Stationary uniform flow solution to the deterministic model}

A particular stationary uniform flow solution to the deterministic model is computed as follows:

- we impose the discharge $Q$ and the Froude number $F$,

$$
F=\frac{|Q|}{H \sqrt{g H}} \Longleftrightarrow H=\left(\frac{Q^{2}}{g F^{2}}\right)^{1 / 3},
$$

which determines the water height $H$. 
- we impose $K_{s}$, then, the equilibrium between the slope and the friction term reads

$$
g H \partial_{x} B=-\frac{\tau}{\rho} \Longleftrightarrow \partial_{x} B=-\frac{Q|Q|}{H^{2} K_{s}^{2} R_{h}^{4 / 3}},
$$

which determines the slope $-\partial_{x} B=\frac{g H F^{2}}{R_{h}^{4 / 3} K_{s}^{2}}$ as a function of $H$ and $F$.

In the present paper, we have used the values presented in the table 1.

\begin{tabular}{|c|cl|}
\hline Parameters & \multicolumn{2}{|c|}{ Values } \\
\hline Domain & {$[0,75]$} & $m$ \\
\hline$l$ & 1 & $m$ \\
\hline$g$ & 9.8 & $m . s^{-2}$ \\
\hline$Q$ & 1 & $m . s^{-1}$ \\
\hline$F$ & 1.5 & $(-)$ \\
\hline$H$ & $\approx 0.36$ & $m$ \\
\hline$U$ & $\approx 2.80$ & $m . s^{-1}$ \\
\hline$R_{h}$ & $\approx 0.21$ & $m$ \\
\hline$-\partial_{x} B$ & $\approx 7$ & $\%$ \\
\hline$K_{s}$ & 30 & $m^{1 / 3} . s^{-1}$ \\
\hline
\end{tabular}

TABLE 1. Initial conditions in torrential regime.

\subsubsection{Description of the stochastic solid flux}

In the previous subsection we described the uniform flow. We now have to describe the characteristic of the solid flux $Q_{s}$ in the Exner equation. We use the stochastic Meyer-Peter-Müller formula (5) and then the definition of the discrete solid flux is

$$
\left(Q_{s}\right)_{i}^{n}=d \sqrt{\frac{g\left(\rho_{s}-\rho\right) d}{\rho}} A_{i}^{n}\left(\left|\left(\tau^{\star}\right)_{i}^{n}\right|-\tau_{c}^{\star}\right)^{\frac{3}{2}} s g\left(\left(\tau^{\star}\right)_{i}^{n}\right)
$$

with the parameters listed in the Table 2.

\begin{tabular}{|c|cl|}
\hline Parameters & \multicolumn{2}{|c|}{ Values } \\
\hline$\rho_{\text {water }}$ & $10^{3}$ & $\mathrm{~kg} . \mathrm{m}^{-3}$ \\
\hline$\rho_{\text {sediment }}$ & $2.65 \times 10^{3}$ & $\mathrm{~kg} \cdot \mathrm{m}^{-3}$ \\
\hline$R$ & $\frac{\rho_{\text {sediment }}-\rho_{\text {water }}}{\rho_{\text {water }}}=1.65$ & $(-)$ \\
\hline$\tau_{c}^{*}$ & 0.047 & $(-)$ \\
\hline$d$ & $10^{-3}$ & $\mathrm{~m}$ \\
\hline$K_{s}$ & 30 & $\mathrm{~m}^{1 / 3} \cdot \mathrm{s}^{-1}$ \\
\hline$A$ & 8 & $(-)$ \\
\hline$\tau^{*}$ & $\approx 8.93$ & $(-)$ \\
\hline
\end{tabular}

TABLE 2. Physical parameters of the sediment flux in torrential regime.

The coefficients $A_{i}^{n}$ are, for $i=0 \cdots N_{x}-1$ and $n=0 \cdots N_{t}$, independent identically distributed random variables with exponential distribution, as suggested by the experimental data. Moreover, the exponential 
probability law is chosen with uniform parameter $\lambda=1 / 8=0.125$, so all coefficients $A_{i}^{n}$ have a mean of $1 / \lambda=8$, their usual deterministic value. Note that the variance then reads $1 / \lambda^{2}=64$.

\subsubsection{Monte Carlo simulations}

For the Monte-Carlo simulations, we use 1000 realizations of the stochastic Saint-Venant-Exner system, which is enough to numerically approximate the first moment of the stochastic quantities by the Central Limit theorem considering the estimated magnitude of the variances (see below). This assertion is made more precise by computing the confidence interval using the empirical variance. The length of this interval turns to be smaller than $10^{-3}$ for all the quantities of interest on the considered meshes, see Table 3 (we are aware that the number of realizations is not sufficient to obtain a very precise evaluation of the second moment of the distributions, but it allows us to correctly approximate the order of magnitude of this quantity with the computed empirical variance). Note also that we choose a final time $T=N_{t} \Delta t$ large enough such that the empirical variance of all the quantities of interest seems close to long-time stationary values as indicated in Figure 2.

\begin{tabular}{|c|c|c|}
\hline & Empirical variance & Size of the confidence interval \\
\hline $\mathrm{B}$ & $\in\left[2 \times 10^{-5}, 2 \times 10^{-4}\right]$ & $\in\left[1.4 \times 10^{-4}, 4.5 \times 10^{-4}\right]$ \\
\hline $\mathrm{U}$ & $\in\left[5 \times 10^{-4}, 4 \times 10^{-3}\right]$ & $\in\left[7.1 \times 10^{-4}, 2 \times 10^{-3}\right]$ \\
\hline
\end{tabular}

TABLE 3. Characterization of the confidence interval
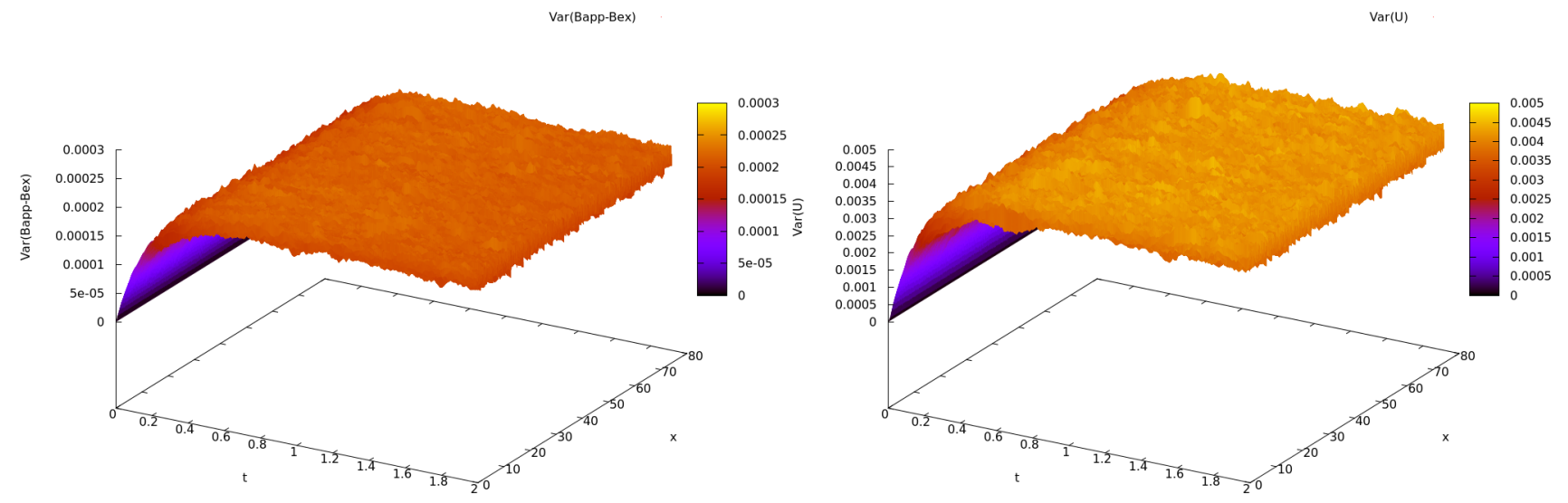

Figure 2. Variance of the bottom topography (left) and of the velocity (right) as a function of time and space for the finest mesh

In all the results, we are not interested in the bottom topogaphy itself but in the deviation of the bottom topography, denoted Bapp on the figures, from its initial value, denoted Bex on the figures (that is also the stationary one for the deterministic case).

We present in Figure 3 the empirical mean of the topography as a function of the abcissa $x$ at the fixed time $T$ for two different meshes $\left(N_{x}=150\right.$ and $\left.N_{x}=2400\right)$, and in Figure 4 the empirical variance of the same quantity for a series of meshes where the number of points varies between the two limits mentioned above. The empirical variance allows us to evaluate the confidence interval and then to check that the oscillations in the empirical mean are not significant. It follows that we can consider the empirical mean is constant in space. Since the empirical mean appears to be centered around zero we can conclude that the mean value of the 
disturbed bottom remains equal to the original topography. This is satisfying: when considering our stochastic Saint-Venant-Exner system, we had in mind to require that the expectation of the physical variables coincide with their deterministic values in equilibrium conditions (such as the stationary uniform flow conditions here).

Moreover, we also observe that the long-time limit of the empirical variance of the stochastic bottom topography seems to converge to a uniform value when we refine the mesh, which also indicates the possibility of a physically-interesting long-time behaviour: the long-time solutions of our stochastic Saint-Venant-Exner system may actually model the uncertainty in the location of the river bed at the grain-scale, i.e. ripples.

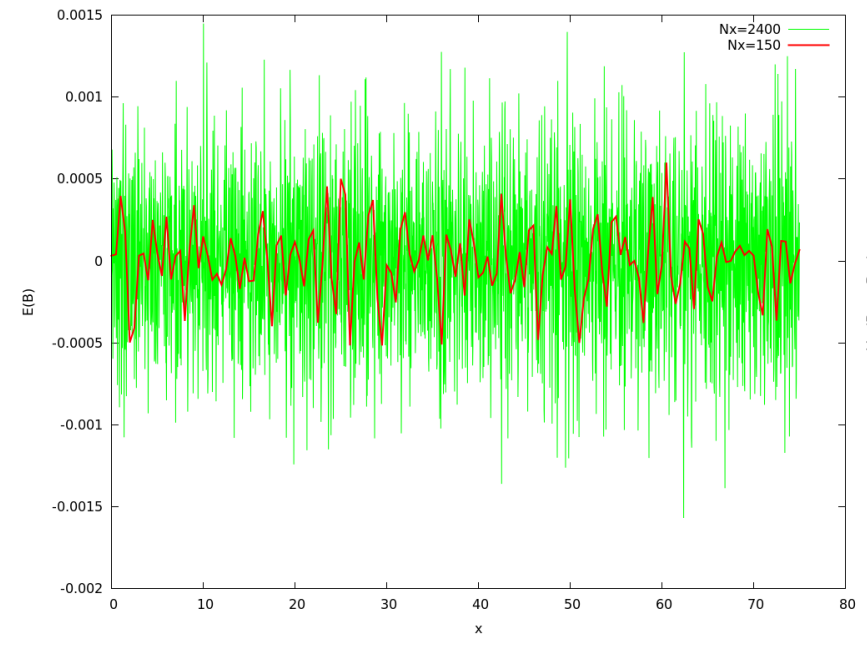

Figure 3. Empirical mean of the topography deviation for two meshes

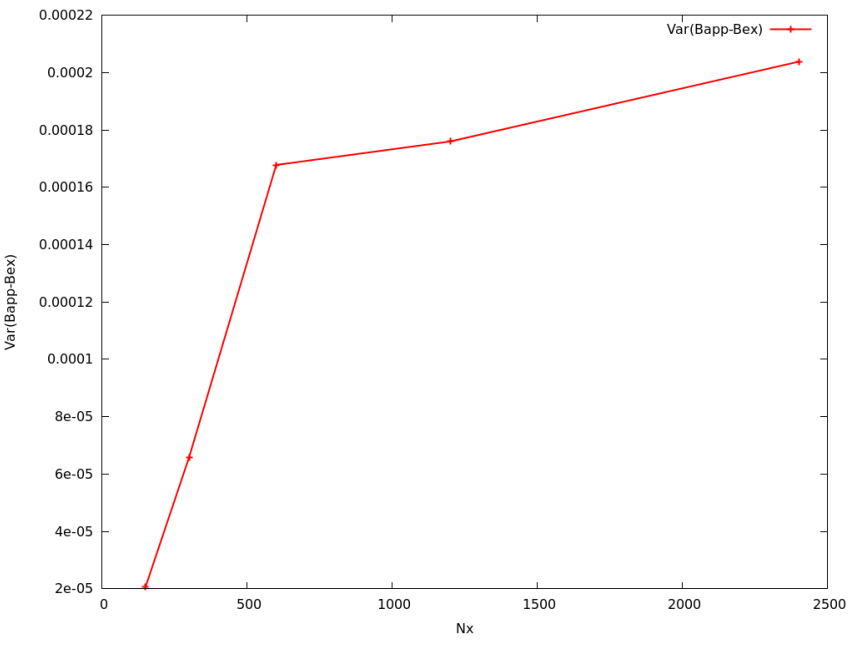

FIGURE 4. Empirical variance of the topography deviation for different meshes

However, let us now turn to the fluid quantities. First, we note that the expectation of the water height and of the bottom topography remain solutions to conservative equations. So the uniform stationary values that $\mathbb{E}(H)$ and $\mathbb{E}(B)$ can be expected to reach at large times in the present periodic setting are necessarily the same as their initial deterministic values. But as concerns the discharge, it holds (recall (6b))

$$
\partial_{t} \mathbb{E}(Q)+\partial_{x} \mathbb{E}\left(\frac{Q^{2}}{H}+\frac{g H^{2}}{2}\right)=-\mathbb{E}\left(g H \partial_{x} B+\frac{\tau}{\rho}\right)
$$

and its total "mass" over the domain is thus not conserved a priori (even in the mean) insofar as the source

$$
\int_{\mathcal{S}} \mathbb{E}\left(g H \partial_{x} B+\frac{\tau}{\rho}\right)
$$

is not zero at all times. (One can see two effects here: i) each trajectory of the stochastic systems departs from equilibrium and has a priori a non-zero spatial average of the source, so nor in the mean for the expectation (13), or ii) nonlinearities imply, for instance, $\mathbb{E}\left(H \partial_{x} B\right) \neq \mathbb{E}(H) \mathbb{E}\left(\partial_{x} B\right)$ even though $\mathbb{E}(H)$ and $\mathbb{E}\left(\partial_{x} B\right)$ have uniformly the initial values of $H$ and $\partial_{x} B$ corresponding to a pointwise-in-space equilibrium with zero source.) Consequently, functions of the velocity or of the discharge, like the energy $Q^{2} / H+g H(H+B)$ for instance, are a priori not conserved either along time by the dynamics of our model, for the same reasons.

In Figures 5 and 6 , we show for the velocity the same curves as for the bottom topography in Figures 3 and 4. Here also, when computing the confidence interval from the empirical variance, it appears that the empirical mean is constant in space. Nevertheless this empirical mean is now sensitive to the mesh size: we present in Figure 7 the empirical mean as a function of time for the 150 elements mesh and in Figure 8 the value of the empirical mean at final time for the whole serie of meshes. It clearly appears that the empirical 
mean decreases when the mesh size $\Delta x$ decreases, and does not clearly seem to converge, at least for the meshes that we consider here. Now, recalling that, when considering the stochastic Saint-Venant-Exner system, one would like the expectation of the physical variables to coincide with their deterministic values in equilibrium conditions (such as the stationary uniform flow conditions investigated here), this is of course not satisfactory.

In the rest of the paper, we try to improve our new model. To this aim, we come back in the next section to a simpler problem where we consider the classical Saint-Venant system with a fixed (i.e. time-independent) topography. The bottom topography however remains stochastic in the sense that we consider (time-independent) random perturbations of the constant-slope deterministic uniform reference flow.

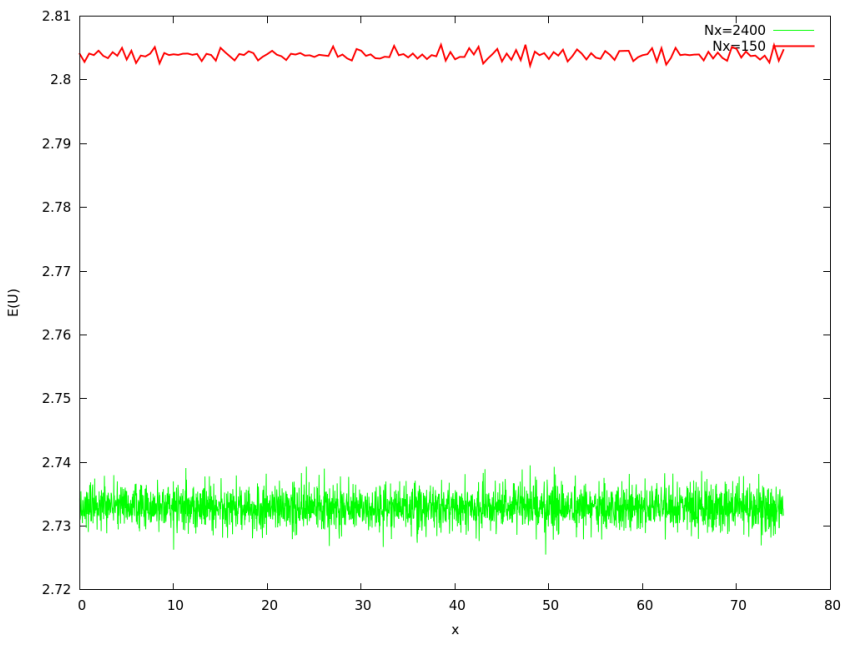

Figure 5. Empirical mean of the velocity for two meshes

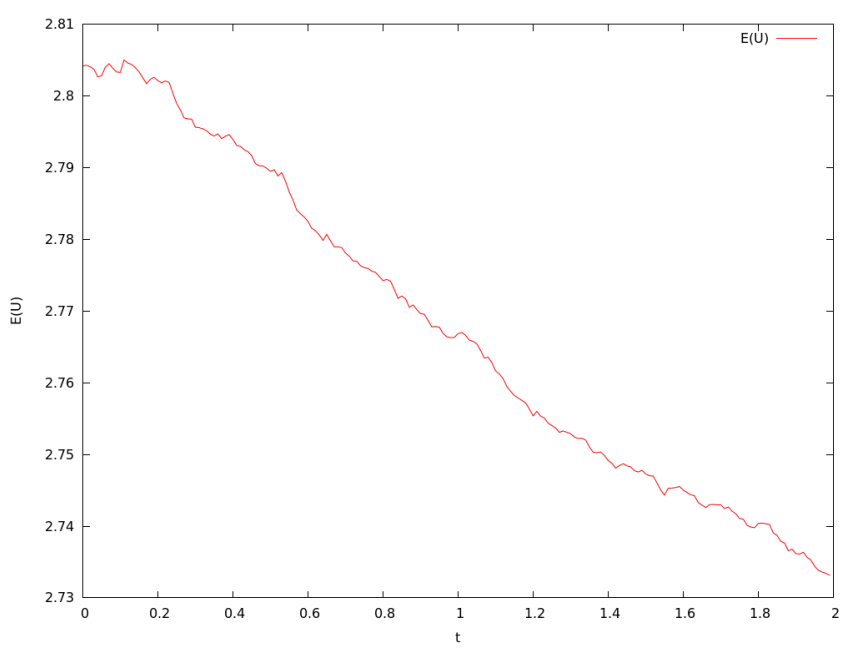

Figure 7. Empirical mean of the velocity as a function of time for the finest mesh

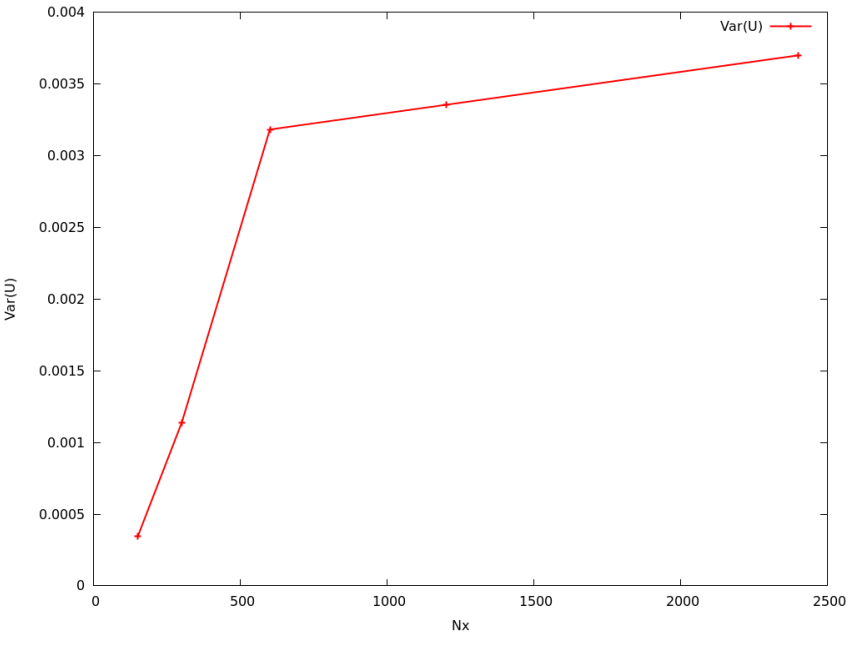

FiguRE 6. Empirical variance of the velocity for different meshes

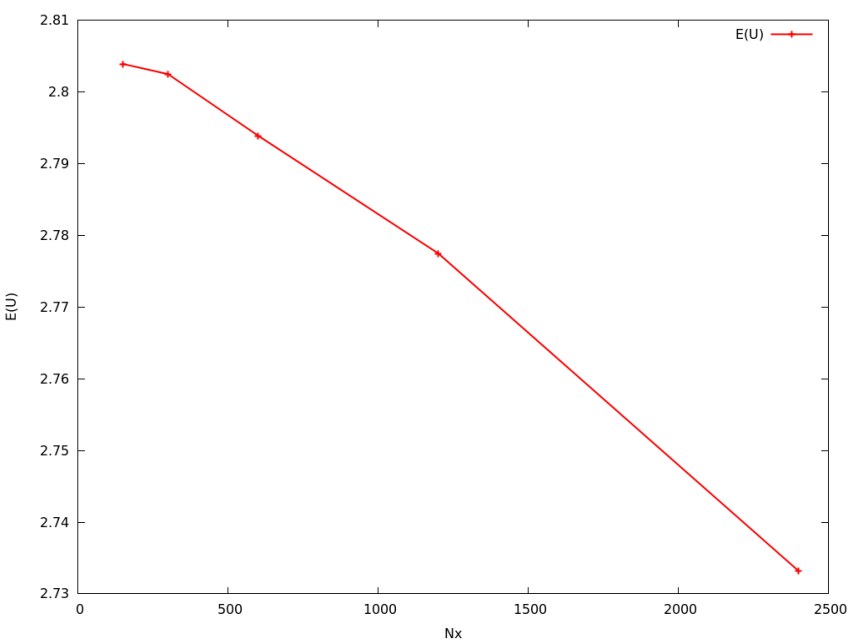

Figure 8. Empirical mean of the velocity for different meshes 
Before turning to the Saint-Venant system, let us present in Figure 9 the probability density functions for the bottom topography and the velocity on the finest mesh. The PDFs are represented at a given point $x$ (previous figures show that the density function, or at least its two first moments, is not sensitive to space) and for some times $t^{n}$. We are aware of the lack of convergence for these PDFs due to the relatively small number of realizations we performed but it seems to us interesting to mention two facts: first, both PDFs appear to be centered around their mean value even if the original noise introduced in the solid flux $Q_{s}$ does not follow a centered density function. Second, the PDF for the velocity is another way to exhibit that its empirical mean decreases when time increases, see Figure 9, but it also appears that the PDF just shifts to the left without large deformation (that is also confirmed by the fact the empirical variance remains constant after a short time, see Figure 6).
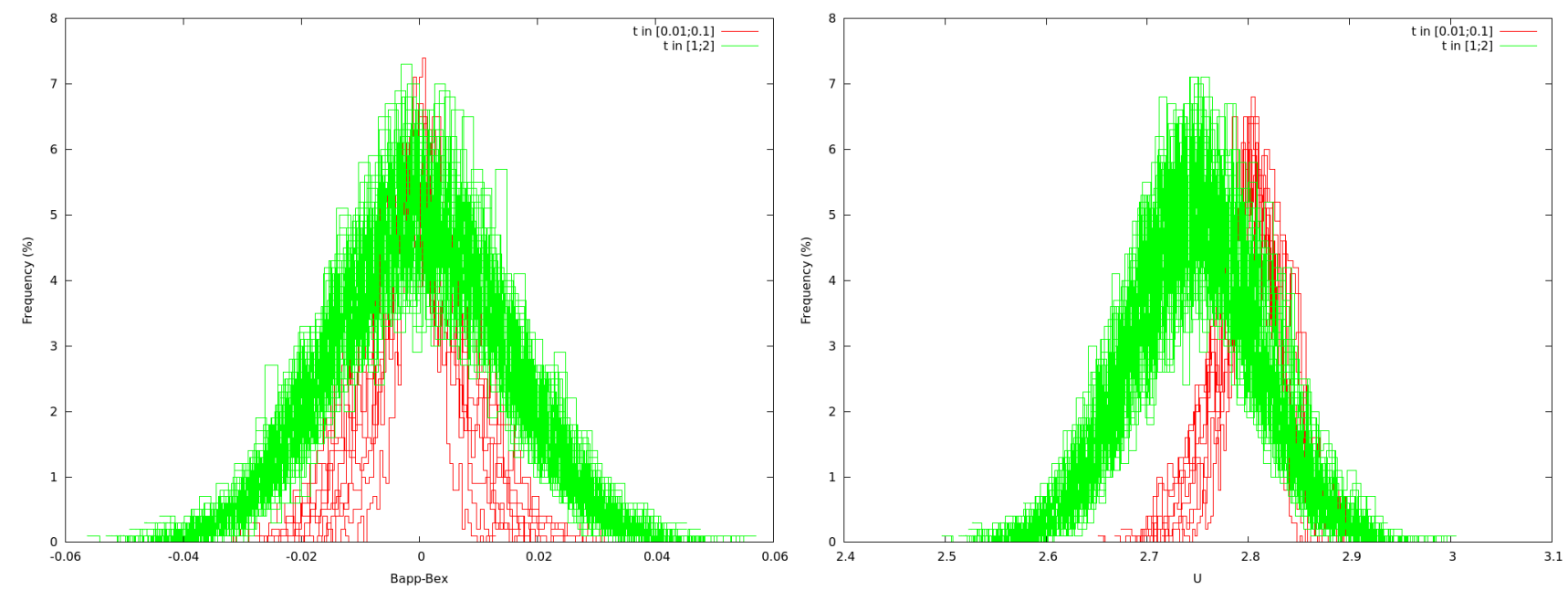

Figure 9. Superimposed PDFs for the topography deviation (left) and the velocity (right) computed on the finest mesh at times $[0.01 ; 0.1]$ and $[1 ; 2]$.

\subsubsection{Stochastic Saint-Venant system}

Currently, we have exhibited a phenomenon of energy diffusion, characterized by the decrease of the velocity, when adding noise to the sediment flux of the Saint-Venant-Exner model. To better understand this phenomenon, we propose in the following to treat another stochastic Saint-Venant system inspired by works done for Burgers equation [5]. Starting from (4), we propose to consider a stochastic bottom topography $B$ defined by

$$
\left\{\begin{array}{l}
B_{i+1 / 2}^{n}=B_{i+1 / 2}^{0}+\tilde{B}_{i+1 / 2} \\
\tilde{B}_{i+1 / 2}=\alpha \sqrt{\Delta x} \sum_{k=1}^{N / 2} \frac{1}{k}\left(a_{k} \cos \left(2 k \pi \frac{i+1 / 2}{N}\right)+b_{k} \sin \left(2 k \pi \frac{i+1 / 2}{N}\right)\right)
\end{array}\right.
$$

where $B_{i+1 / 2}^{0}$ corresponds to the non-perturbed initial bottom topography, $a_{k}$ and $b_{k}$ are random variables following a normal law $\mathcal{N}(0,1)$, and $\alpha$ is an imposed amplitude. The process consists on disturbing the bottom topography $B$ at the initial state $(t=0)$ for each realization. It clearly appears that each coefficient of the added perturbation follows a normal law $\mathcal{N}\left(0, \alpha^{2} \Delta x\right)$. With the whole expression of noise, we consider a particular kind of spatial correlation which would formally tend to a white noise when the mesh size tends to zero.

We consider again a torrential flow over a constant sloped bottom topography and the initial parameters are given in Table 1. One recalls that we impose periodic limit conditions at the boundaries of the domain. The 
different simulations are done with a parameter of amplitude $\alpha$ equal to $10^{-1}$.

The framework of the test case is the same as the Saint-Venant-Exner case; we work with a 150 points mesh and also simulate 1000 realizations. Given the fact that the bottom topography does not evolve in time, we exhibit the range of values of the injected noise for two realizations in Figure 10. The values oscillate in an interval of length of the order of $10^{-1}$ which is in accordance with the imposed value of the amplitude of the noise.

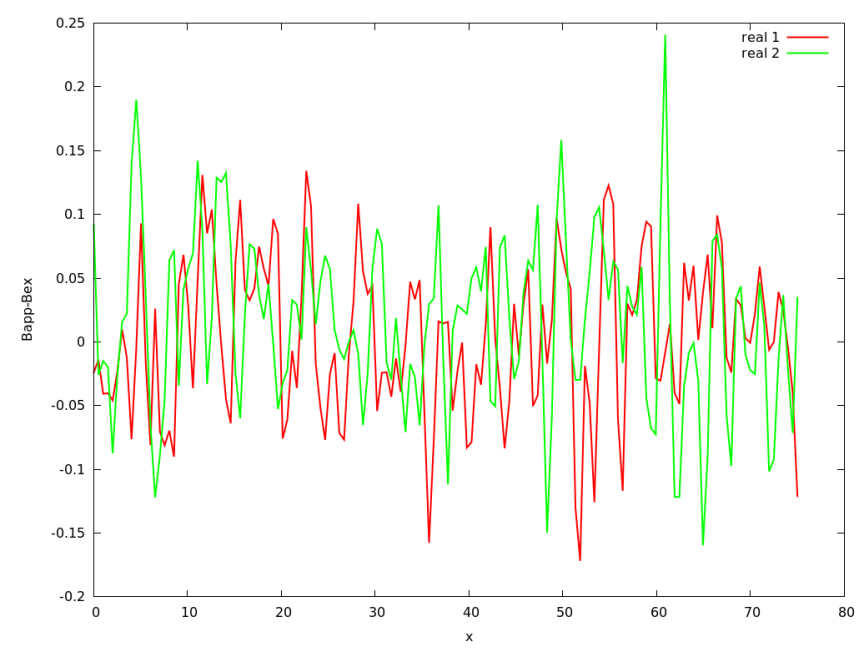

Figure 10. Added noise as a function of space with $\alpha=10^{-1}$ and $N_{x}=150$ for two different realizations

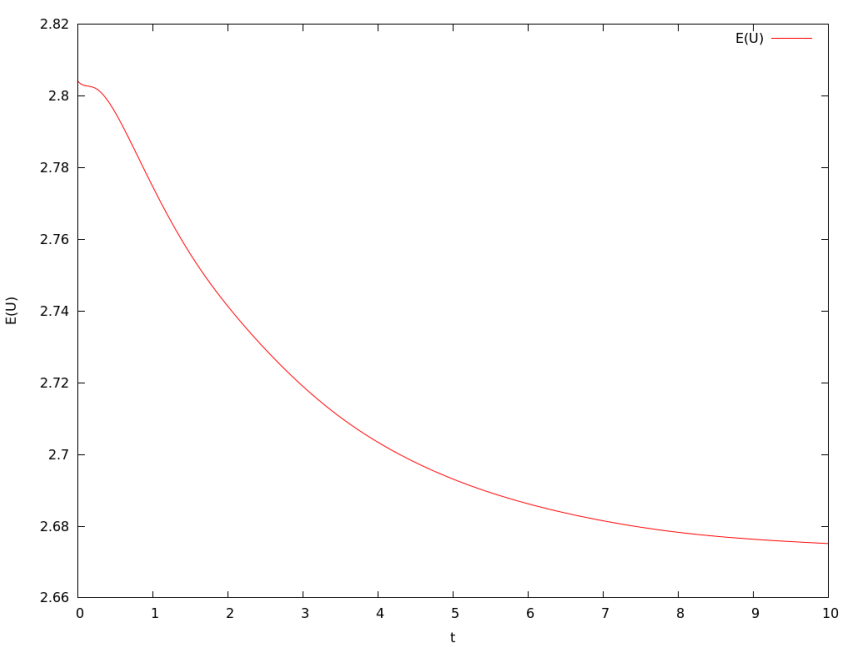

Figure 11. Empirical mean of the velocity as a function of time

Concerning the hydraulic part, we present the respective variances of the water height $H$ and the velocity $U$ in Figure 12. The final values are not the same as in the previous case due to the difference between the range of values taken by the injected noise in the present case and in the Saint-Venant-Exner case. In spite of this observation, the behavior of these curves is similar to the previous ones observed for the Saint-VenantExner system. The important fact is that the empirical mean of the velocity decreases as a function of time to a new uniform value, see Figure 11. This result is in accordance with the result obtained for the stochastic Saint-Venant-Exner model. For information purposes only, the PDF of the water height and the velocity are presented on Figure 13 on which we can also note the shift previously mentioned for the PDFs of the velocity. (Note that in this new configuration, the PDFs of the fluid quantities are skewed even if the PDF of the bottom topography is symmetric.)

Now, since the decrease of the discharge (and thereby the energy loss) is due to the imbalance between the slope and the friction terms, we suggest to choose, in the stochastic Saint-Venant models, a Strickler coefficient $K_{s}$ different from the one in the deterministic case. But we propose to proceed in the same spirit as in the deterministic case: the Strickler coefficient $K_{s}$ should in fact be calibrated so as to enforce the equilibrium (12).

In the following, we compute the new coefficient at each time step as a deterministic function of moments of $H$ and $Q$. To establish an expression, at the discrete level, we require an equality between the expectation of the sum in space of $Q_{i}^{n}$ and $Q_{i}^{n+1}$

$$
\mathbb{E}\left[\sum_{i=0}^{N_{x}-1} Q_{i}^{n+1}\right]=\mathbb{E}\left[\sum_{i=0}^{N_{x}-1} Q_{i}^{n}\right]
$$

thus imposing that no energy is dissipated by the model. In that way, it is possible to express the Strickler coefficient as a time dependent deterministic coefficient $\left(K_{s}\right)^{n}$, which can be used explicitly for every realization 

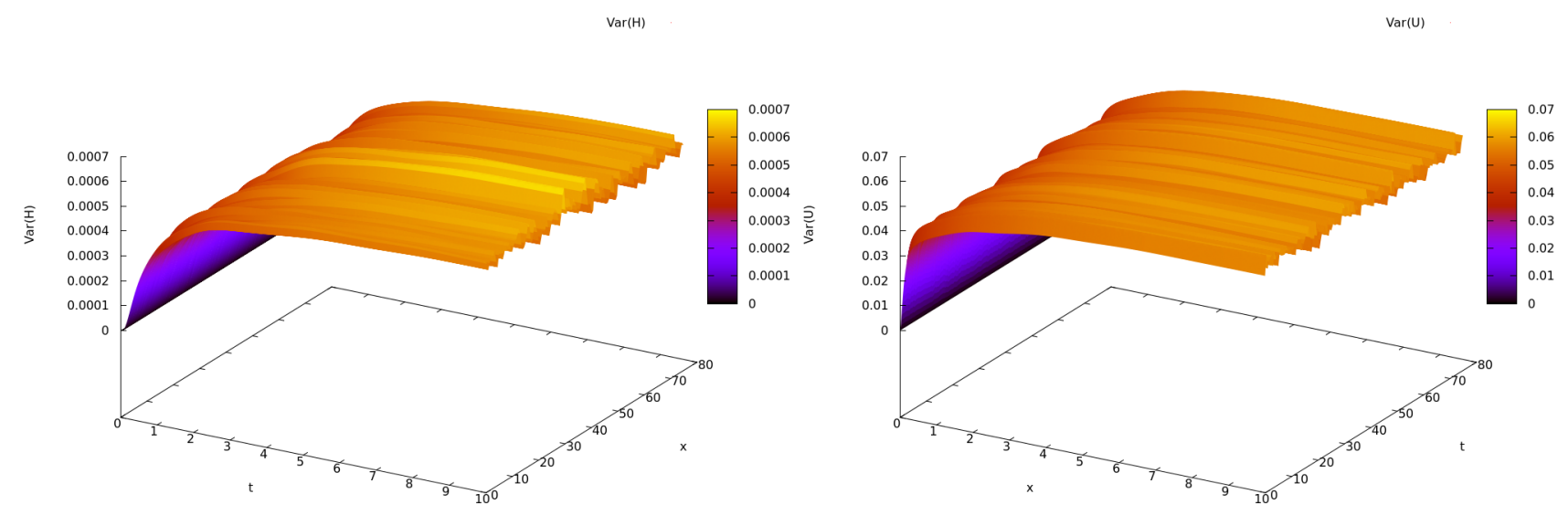

FIGURE 12. Variance of the water height (left) and the velocity (right) as a function of space and time for $N_{x}=150$

$\omega$ and each time step $t^{n}$ in the momentum conservation equation of (7). Precisely, we obtain the formula

$$
\left(K_{s}\right)^{n}=\left(-\frac{\mathbb{E}\left[\sum_{i=0}^{N_{x}-1} \frac{Q_{i}^{\omega, n}\left|Q_{i}^{\omega, n}\right|}{H_{i}^{\omega, n}\left(R_{h, i}^{\omega, n}\right)^{4 / 3}}\right]}{\left(-\partial_{x} B^{0}\right) \times \mathbb{E}\left[\sum_{i=0}^{N_{x}-1} H_{i}^{\omega, n}\right]+\frac{1}{\Delta x} \mathbb{E}\left[\sum_{i=0}^{N_{x}-1} H_{i}^{\omega, n}\left(\tilde{B}_{i+1 / 2}^{\omega}-\tilde{B}_{i-1 / 2}^{\omega}\right)\right]}\right)^{1 / 2}
$$

where we recall that the expectation of the stochastic bottom slope $-\partial_{x} B^{0}$ coincides with the slope (constant and uniform) of the deterministic equilibrium.

The new stochastic system (7) is now nonlinear in the sense of McKean (the coefficients are functions of the stochastic process), and much more difficult to handle numerically (all realizations in a Monte-Carlo simulation need communicating one-another). Furthermore, it is not clear whether requiring equilibrium through the mean-field approximation above will actually be enough for the long-time expectations in the stochastic SaintVenant-Exner system to coincide with their deterministic counterparts at equilibrium. We postpone these questions to future works and limit here to the simplified stochastic Saint-Venant system.

For this system, and starting from the deterministic equilibrium where $K_{s}=30$, one actually maintains an equilibrium "in the empirical mean" with the formula (14) after initially perturbing the bottom topography. We present in Figure 15 the evolution in time of the empirical mean of $U$ for Monte-Carlo simulations. It remains close to the equilibrium. We also exhibit in Figure 14 that $K_{s}$ converges fast in time to a new constant value. This value is higher than the deterministic equilibrium value of $K_{s}$ equal to 30 (around 31.4).

This higher value is consistent with the fact that the introduction of a friction coefficient in the Saint-Venant system is often presented as a way to take into account (among other phenomena) a roughness of the topography explicitly introduced in the model, with a view to maintaining uniform flow conditions. On the contrary, when some additional roughness is explicitly included through (stochastic) perturbations of the bottom topography, the parameterized friction effect can decrease, and $K_{s}$ increase.

The fact that the new Strickler coefficient can adapt fast to perturbations of the bottom topography in order to maintain an equilibrium is also very promising for the construction of a consistent stochastic Saint-VenantExner model that maintains in the mean the same stationary uniform flows as in the deterministic case. In particular, we have also computed the case where the Strickler coefficient is fixed (time-independent) but with 
value 31.43, from the long-time limit of the previous time-dependent case. Then, the new equilibrium state close to the deterministic equilibrium is still maintained in the mean for large times, as shown in Figure 16. In addition, as expected, the discrete source term is distributed around the value zero, see Figure 17. (Discretely, the friction term obtained with the new Strickler coefficient compensates well the part of the source term related to the slope of the bottom topography in the mean.)
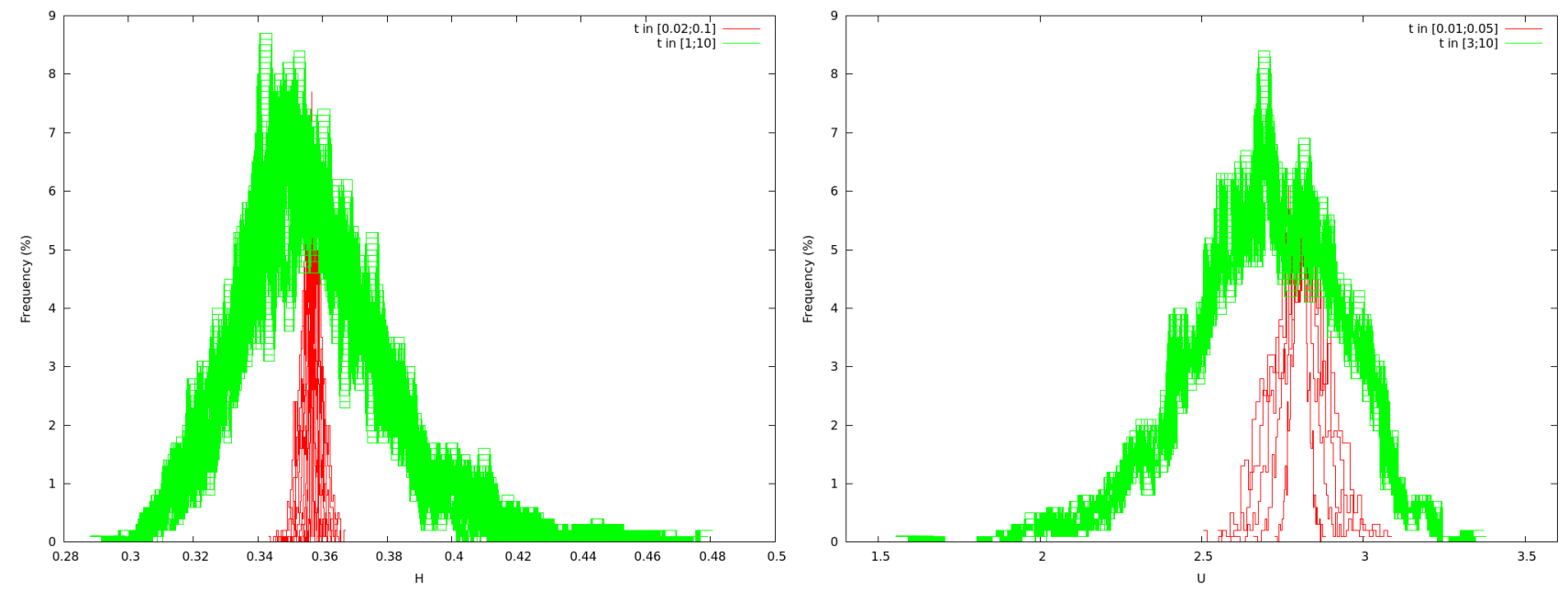

FIGURE 13. Superimposed PDFs for the water height at times $[0.02 ; 0.1]$ and $[1 ; 10]$ (left), and the velocity at times $[0.01 ; 0.05]$ and $[3 ; 10]$ (right) with $N_{x}=150$.

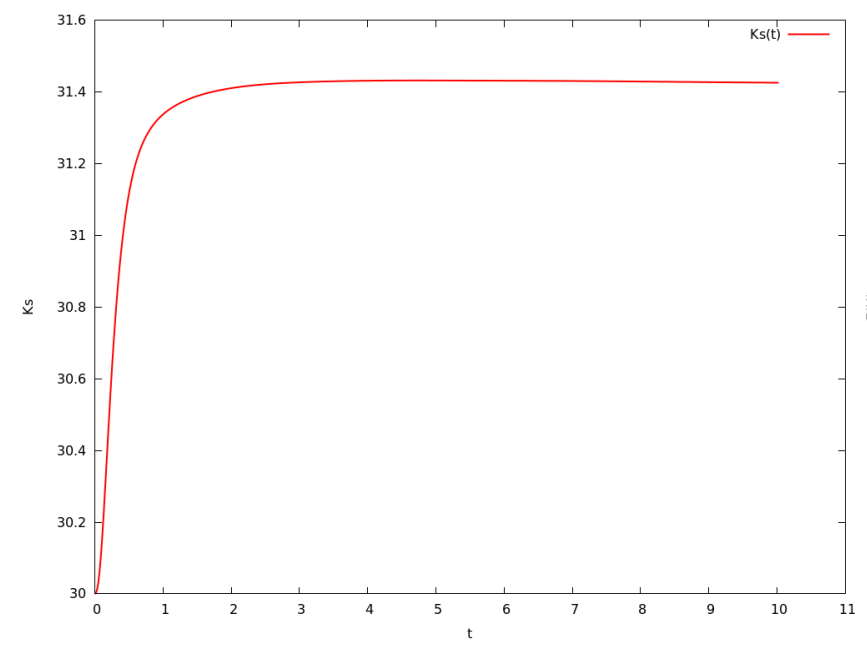

Figure 14. Values of $K_{s}$ as a function of time

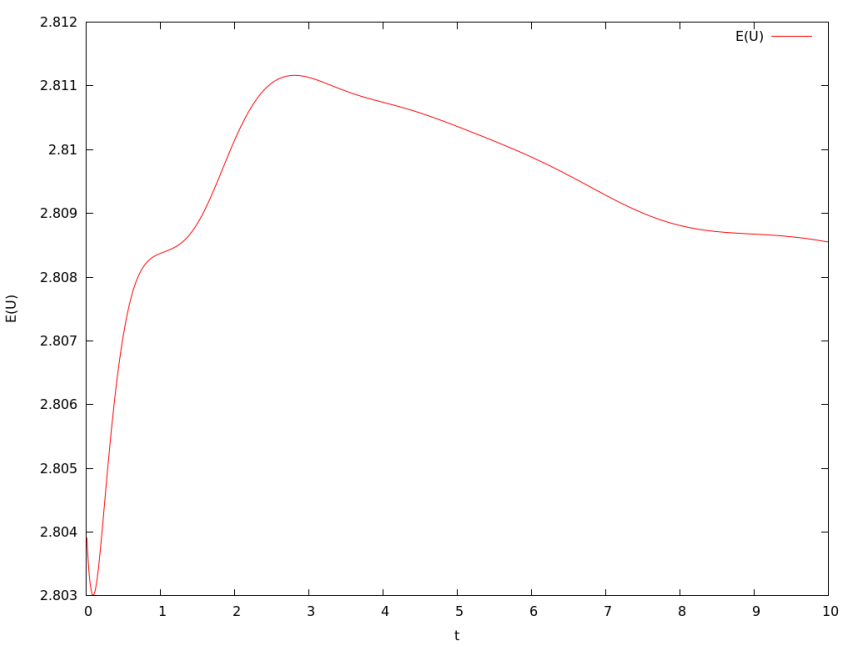

Figure 15. Empirical mean of the velocity as a function of time 


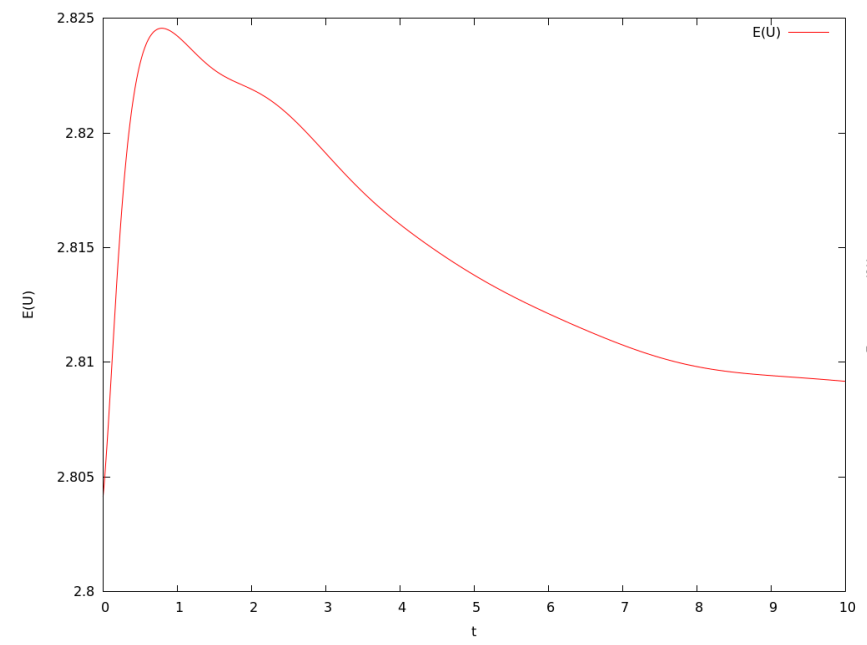

Figure 16. Empirical mean of the velocity as a function of time with $K_{s}=$ 31.43

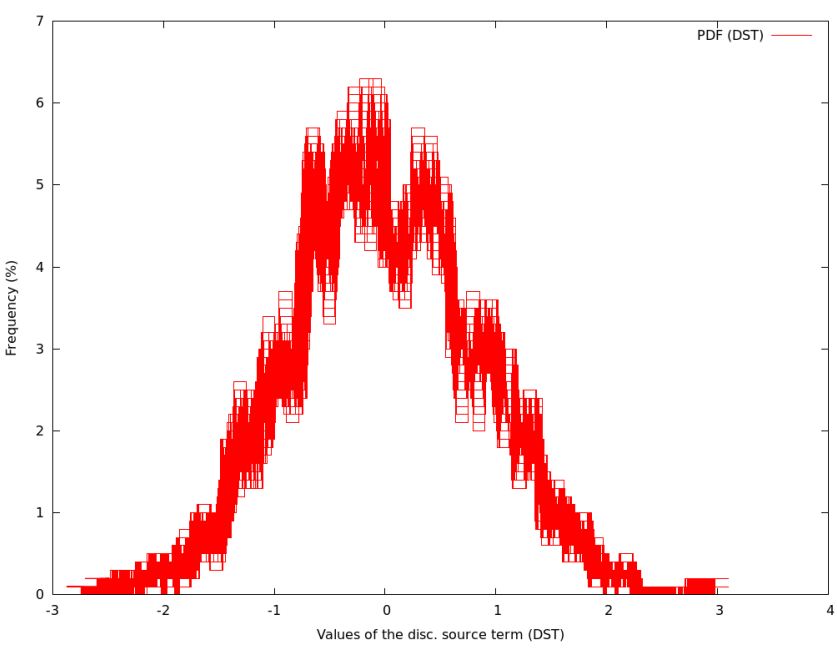

Figure 17. PDF of the discrete source term with $K_{s}=31.43$

Last, let us again present the variance and the PDFs for the water height and the velocity when replacing the constant value of $K_{s}$ previously used (in every test case, $K_{s}=30 \mathrm{~m}^{1 / 3} . \mathrm{s}^{-1}$ ) by a time dependent one as expressed earlier (14). These results are respectively shown in Figures 18 and 19. Compared to the results on Figure 12, those on Figure 18 show that the variance for the water depth and the velocity has overall the same behaviour than the former ones. Moreover, the modified Strickler coefficient has corrected perforce the shift previously observed both for the Saint-Venant-Exner model and for the Saint-Venant system: not only $\mathbb{E}(H)$ and $\mathbb{E}(Q)$ assume uniform values equal to the initial equilibrium conditions for large times, but also the velocity $U=Q / H$, as shown on Figure 15 (in spite of the nonlinearity). (The empirical mean of the velocity at given $x$ yields an approximation of the expectation in an interval with magnitude of order between $10^{-3}$ and $10^{-2}$ at any time.) Moreover, in the PDFs for the velocity shown at given $x$ and various times in Figure 19, it appears that the most probable values is the same at any time.

\begin{tabular}{|l|c|c|c|}
\hline & \multicolumn{2}{|c|}{ Saint-Venant } & Saint-Venant-Exner \\
\hline & with constant $K_{s}$ & with modified $K_{s}$ & stochastic $Q_{s}$ \\
\hline$E($ Bapp - Bex $)$ & 0 & 0 & 0 \\
\hline $\operatorname{Var}(B a p p-B e x)$ & $4.10489 \times 10^{-3}$ & $4.10489 \times 10^{-3}$ & $4.3123 \times 10^{-5}$ \\
\hline$E(U)$ & 2.6 & 2.8 & 2.80 \\
\hline $\operatorname{Var}(U)$ & $5.61674 \times 10^{-2}$ & $5.36315 \times 10^{-2}$ & $7.17021 \times 10^{-4}$ \\
\hline$E(H)$ & 0.35 & 0.35 & 0.356 \\
\hline $\operatorname{Var}(H)$ & $5.70804 \times 10^{-4}$ & $4.98172 \times 10^{-4}$ & $6.78487 \times 10^{-6}$ \\
\hline
\end{tabular}

TABLE 4. Comparison of the results obtained for the three test cases with $N_{x}=150$ : SaintVenant + perturbed $B$ and constant $K_{s}$, Saint-Venant + perturbed $B$ and modified $K_{s}$ at each time step, and stochastic Saint-Venant-Exner.

Since we are now able to maintain in the mean the deterministic flow, it is also possible to study the variance of the fluid quantities as a function of the amplitude of the injected noise. As the amplitude of the noise grows, the variance of the velocity $U$ increases. It appears that the square root of the variance of $U$ has a linear 

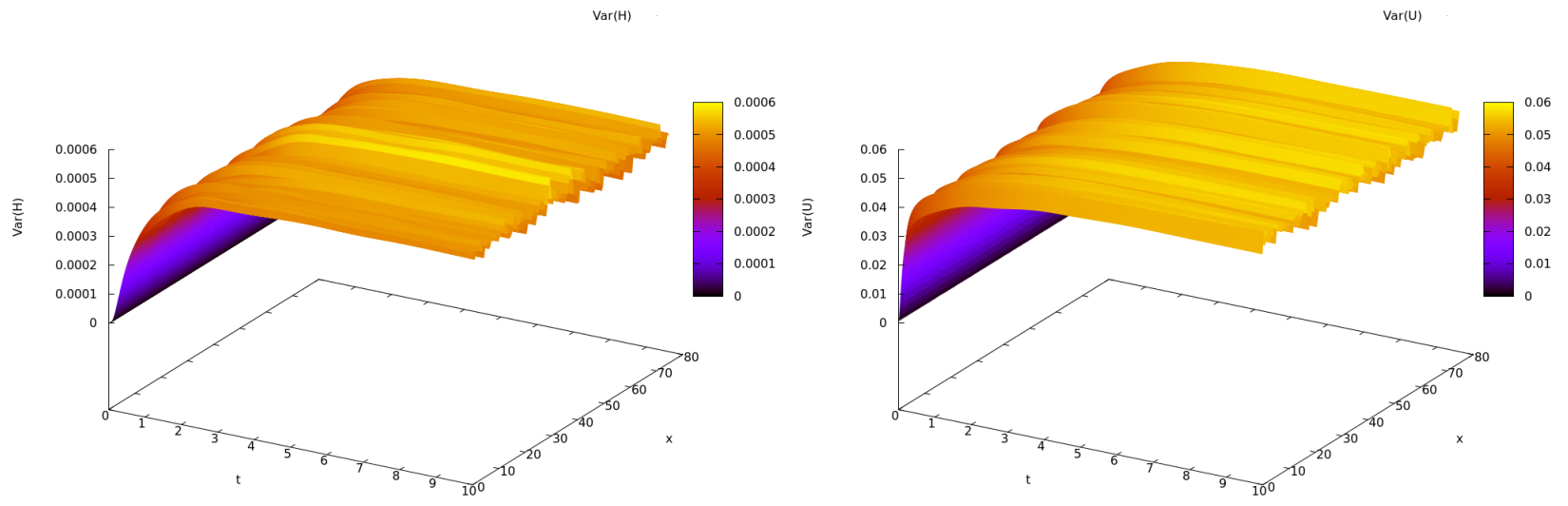

FIGURE 18. Variance of the water height (left) and the velocity (right) as a function of space and time for $N_{x}=150$
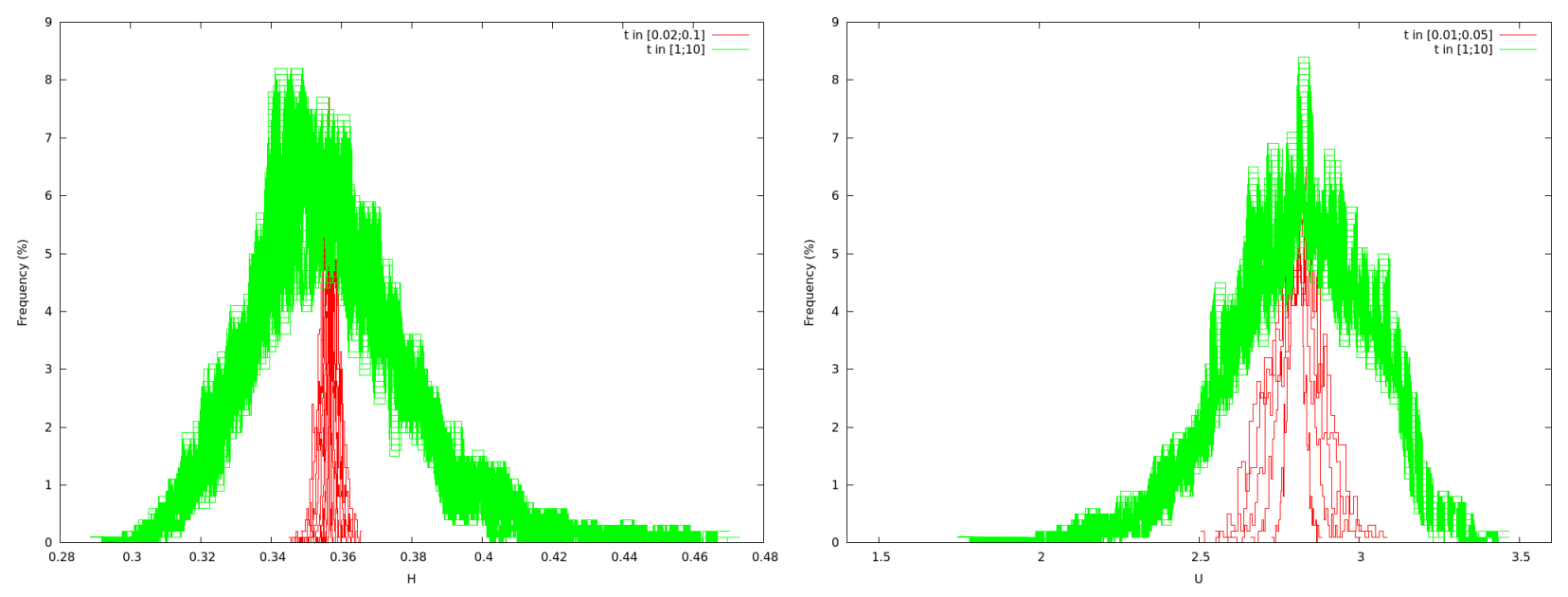

Figure 19. Superimposed PDFs for the water height at times $[0.02 ; 0.1]$ and $[1 ; 10]$ (left), and the velocity at times $[0.01 ; 0.05]$ and $[3 ; 10]$ (right) with $N_{x}=150$.

dependency on the amplitude of the noise $\alpha$ as shown on Figure 20. The slope of the curve is quite constant for amplitudes of noise lower than $\alpha=0.1$ and increases for amplitudes exceeding this last value.

\section{Conclusion}

We have proposed a stochastic version of Saint-Venant-Exner model in order to account for the fluctuations of the solid flux observed experimentally in stationary river beds. We have observed numerically that, in the mean, a naive introduction of noise in the standard Saint-Venant-Exner model generates additional dissipative effects compared with the deterministic case, which is a bad news if one wants the expectation of the stochastic model to coincide with the deterministic one, at least in equilibrium conditions (i.e. stationary uniform flows). Then, the focus was placed on the question of the convergence of this dissipation in fonction of the size of the mesh. We have observed that the more refined the mesh is, the more dissipation there is. The next question 


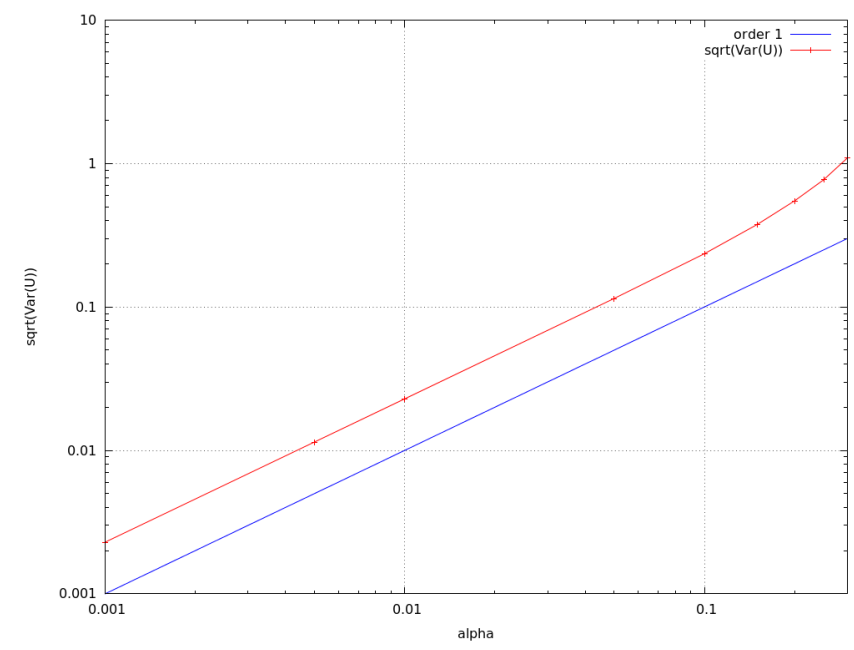

FIGURE 20. Log-log graph of the square root of the variance of $U$ as a function of the amplitude of the noise $\alpha$

is thus how to better model the noise introduced in Saint-Venant-Exner model so as to compensate for the dissipation introduced. In particular, the scaling of the injected noise with respect to time and space was very naive. One alternative model could be based on better correlating the space-time increments of some random variables in the system. This is a difficult mathematical task, all the more when it is a priori without physical data. As an alternative, we have thus proposed a numerical method to correct, at discrete level, the undesired added dissipation.

We have suggested to define the Strickler coefficient in a stochastic model so as to maintain exactly in the mean equilibrium conditions that are known a priori. In the case of our finite-volume numerical scheme, the new Strickler coefficient can be computed explicitly, as a function of the whole stochastic process. Though, this modification makes the proposed stochastic Saint-Venant-Exner model quite nonlinear, and more difficult to simulate. In this work, we have thus only checked yet, numerically, that once the bottom topography is perturbed initially (but not evolving in time yet, i.e. a simplified stochastic model), the new Strickler coefficient actually makes sense, and compensates fast enough the mean diffusion due to the stochastic perturbations of the bottom topography.

It remains to see i) whether it is still possible to maintain the deterministic equilibrium in the mean when the bottom topography is perturbed in time in a stochastic Saint-Venant-Exner model, ii) whether the new model is robust as concerns convergence to a continuous time-space model, and iii) the kind of physical information that can actually be supported by the stochastic variable $A$ (characteristic size of ripples...) compared with other ones, e.g. $\tau^{\star}$.

\section{Acknowledgements}

The authors thank A. Recking for the experimental data. The authors greatly thank the CIRM for its warm welcome during the CEMRACS. EA and PU thank EDF R\&D and Laboratoire d'Hydraulique Saint-Venant for financially supporting their participation to CEMRACS 2013.

\section{REFERENCES}

[1] B. Alvarez-Samaniego and P. Azérad. Existence of travelling-wave solutions and local well-posedness of the Fowler equation. Discrete Contin. Dyn. Syst. Ser. B, 12(4):671-692, 2009. 
[2] C. Ancey, A. Davison, T. Böhm, M. Jodeau and P. Frey. Entrainment and motion of coarse particles in a shallow stream down a steep slope. Journal of Fluids Mechanics, 595, 2008.

[3] C. Ancey. Stochastic modeling in sediment dynamics: Exner equation for planar bed incipient bed load transport conditions. J. Geophys. Res., 115:1-21, 2010.

[4] E. Audusse, C. Berthon, C. Chalons, O. Delestre, I. Giesselman, M. Goutal, N. Jodeau, G. Sadaka and J. Sainte-Marie. Sediment transport modelling : Relaxation schemes for Saint-Venant-Exner and three layer models. ESAIM: Proc., CEMRACS 2011, 38:78-98, 2012.

[5] E. Audusse, S. Boyaval, Y. Gao and D. Hilhorst. Numerical simulations of the periodic inviscid Burgers equation with stochastic forcing. ESAIM: Proc., CEMRACS 2013.

[6] F. Ballio, V. Nikora and S. E. Coleman. On the definition of solid discharge in hydroenvironment research and applications. J. Hydraul. Res., 2014

[7] P. Claudin B. Andreotti and S. Douady. Selection of dune shapes and velocities. part 2: A two-dimensional modelling. Eur. Phys. J. B, 28:341, 2002.

[8] R. A. Bagnold. The nature of saltation and of 'bed-load' transport in water. Proceedings of the Royal Society of London. A. Mathematical and Physical Sciences, 332(1591):473-504, 1973.

[9] F. Ballio, V. Nikora and S. E. Coleman. On the definition of solid discharge in hydro-environment research and applications. J. Hydraul. Res., 52(2):173-184, 2014.

[10] M. J. Castro Díaz, E. D. Fernández-Nieto, and A. M. Ferreiro. Sediment transport models in shallow water equations and numerical approach by high order finite volume methods. Comput. \& Fluids, 37(3):299-316, 2008.

[11] O. Devauchelle, L. Malverti, E. Lajeunesse, P.-Y. Lagrée, C. Josserand and K.-D. Nguyen Thu-Lam. Stability of bedforms in laminar flows with free surface: from bars to ripples. J. Fluid Mech., 642:329-348, 2010.

[12] H. Einstein. The bed-load function for sediment transportation in open channel flows. Technical Report 1026, United States Department of Agriculture, 1950.

[13] K. El Kadi Abderrezzak and A. Paquier. One-dimensional numerical modeling of sediment transport and bed deformation in open channels. Water Resources Research, 45, 2009.

[14] F. M. Exner. Über die wechselwirkung zwischen wasser und geschiebe in flüssen. Akad. Wiss. Wien Math. Naturwiss. Klasse, 134(2a):165-204, 1925.

[15] R. Fernandez Luque and R. Van Beek. Erosion and transport of bed-load sediment. J. Hydraul. Res., 14(2):127-144, 1976.

[16] E. Foufoula-Georgiou and C. Stark. Introduction to special section on stochastic transport and emergent scaling on earth's surface: Rethinking geomorphic transport - stochastic theories, broad scales of motion and nonlocality. J. Geophys. Res.: Earth Surface, 115(F2), 2010.

[17] J. R. D. Francis. Experiments on the motion of solitary grains along the bed of a water-stream. Proceedings of the Royal Society of London. A. Mathematical and Physical Sciences, 332(1591):443-471, 1973.

[18] D. J. Furbish, A. E. Ball and M. W. Schmeeckle. A probabilistic description of the bed load sediment flux: 4. fickian diffusion at low transport rates. J. Geophys. Res., 117, 2012.

[19] D. J. Furbish, J. C. Roseberry, and M. W. Schmeeckle. A probabilistic description of the bed load sediment flux: 3. the particle velocity distribution and the diffusive flux. J. Geophys. Res., 117, 2012.

[20] D. J. Furbish, P. K. Haff, J. C. Roseberry, and M. W. Schmeeckle. A probabilistic description of the bed load sediment flux: 1. theory. J. Geophys. Res., 117, 2012.

[21] D. J. Furbish and M. W. Schmeeckle. A probabilistic derivation of the exponential-like distribution of bed load particle velocities. Water Resour. Res., 49(3):1537-1551, 2013.

[22] V. Ganti, A. Singh, P. Passalacqua and E. Foufoula-Georgiou. Subordinated brownian motion model for sediment transport. Phys. Rev. E, 80(1), 2009.

[23] G. Garegnani, G. Rosatti and L. Bonaventura. Free surface flows over mobile bed: mathematical analysis and numerical modeling of coupled and decoupled approaches. Communications in Applied and Industrial Mathematics, 2(1), 2011.

[24] G. Garegnani, G. Rosatti and L. Bonaventura. On the range of validity of the exner-based models for mobile-bed river flow simulations. J. Hydraul. res., 2013.

[25] W. Graf and M. Altinakar. Hydraulique fluviale Tome 2 Ecoulement non permanent et phénomènes de transport. Presses polytechniques et universitaire romandes, 1996.

[26] P. Hall. Alternating bar instabilities in unsteady channel flows over erodible beds. J. Fluid Mech., 499:49-73, 2004.

[27] J.-M. Hervouet. Hydrodynamics of free surface flows. Modelling with the finite element method. Wiley \& Sons, 2007.

[28] T.-J. Hsu, J. T. Jenkins and P. L.-F. Liu. On two-phase sediment transport: sheet flow of massive particles. Proceedings of the Royal Society of London. Series A: Mathematical, Physical and Engineering Sciences, 460(2048):2223-2250, 2004.

[29] J. T. Jenkins and D. Berzi. Kinetic theory applied to inclined flows. Granular Matter, 14(2):79-84, 2012.

[30] D. J. Jerolmack and D. Mohrig. A unified model for subaqueous bed form dynamics. Water Resour. Res., 41, 2005.

[31] M. Kleinhans and L. van Rijn. Stochastic prediction of sediment transport in sand-gravel bed rivers. J. Hydraul. Eng., 128(4):412-425, 2002.

[32] E. Lajeunesse, L. Malverti and F. Charru. Bed load transport in turbulent flow at the grain scale: Experiments and modeling. J. Geophys. Res., 115, 2010. 
[33] E. Meyer-Peter and R. Müller. Formulas for bedload transport. Proceedings, Second Congress IAHR, Stockholm, Sweden, 1948.

[34] Y. Niño, A. Atala, M. Barahona and D. Aracena. Discrete particle model for analyzing bedform development. J. Hydraul. Eng., 128(4):381-389, 2002.

[35] M. Ouriemi, P. Aussilous and E. Guazzelli. Sediment dynamics. part 1. bed-load transport by laminar shearing flows. J. Fluid Mech., 636:295-319, 2009.

[36] M. Ouriemi, P. Aussilous and E. Guazzelli. Sediment dynamics. part 2. dune formation in pipe flow. J. Fluid Mech., 636:321336, 2009.

[37] A. S. Paintal. A stochastic model of bed load transport. J. Hydraul. Res., 9(4):527-554, 1971.

[38] A. N. Papanicolaou, M. Elhakeem, G. Krallis, S. Prakash and J. Edinger. Sediment Transport Modeling Review-Current and Future Developments. J. Hydraul. Eng., 2008.

[39] G. Parker. Surface-based bedload transport relation for gravel rivers. J. Hydraul. Res., 28(4):417-436, 1990.

[40] G. Parker, C. Paola and S. Leclair. Probabilistic Exner sediment continuity equation for mixtures with no active layer. J. Hydraul. Eng., 126:818-826, 2000.

[41] A. Recking, P. Frey, A. Paquier, P. Belleudy and J.-Y. Champagne. Bed-Load Transport Flume Experiments on Steep Slopes. J. Hydraul. Eng., 134(9), 2008.

[42] J. C. Roseberry, M. W. Schmeeckle and D. J. Furbish. A probabilistic description of the bed load sediment flux: 2. particle activity and motions. J. Geophys. Res., 117, 2012.

[43] A. Shields. Application of similarity principles and turbulence research to bed-load movement. PhD thesis, California Institute of Technology , Pasadena, CA, Soil Conservative Service, Cooperative Laboratory, 1936. Translated by W.I. Ott and J.C. van Uchelen from: Anwendung der Aehnlichkeitsmechanilr und der Turbulenzforschung auf die Geschiebe bewegung, Mitteilungen der Preussischen Versuchsanstalt fuer Wasserbau und Schiffbau, Berlin, 1936.

[44] C. Swartenbroekx, Y. Zech and S. Soares-Frazão. Two-dimensional two-layer shallow water model for dam break flows with significant bed load transport. Int. J. Numer. Meth. Fl., 73(5):477-508, 2013.

[45] C. Villaret, J.-M. Hervouet, R. Kopmann and U. Merkel. Morphodynamic modeling using the Telemac finite-element system. Computers and Geosience, 53, 2013.

[46] F. Wu and Y. Chou. Rolling and lifting probabilities for sediment entrainment. J. Hydraul. Eng., 129(2):110-119, 2003.

[47] D.-Y. Zhong, G.-Q. Wang and L. Zhang. A bed-load function based on kinetic theory. International Journal of Sediment Research, 27(4):460-472, 2012. 